\title{
All-Optical Quantum Information Processing Using Rydberg Gates
}

\author{
D. Paredes-Barato* and C. S. Adams ${ }^{\dagger}$ \\ Joint Quantum Centre (JQC) Durham-Newcastle, Department of Physics, Durham University, \\ Rochester Building, South Road, Durham DH1 3LE, United Kingdom
}

(Received 1 October 2013; published 28 January 2014)

\begin{abstract}
In this Letter, we propose a hybrid scheme to implement a photonic controlled- $z$ (CZ) gate using photon storage in highly excited Rydberg states, which controls the effective photon-photon interaction using resonant microwave fields. Our scheme decouples the light propagation from the interaction and exploits the spatial properties of the dipole blockade phenomenon to realize a CZ gate with minimal loss and mode distortion. By excluding the coupling efficiency, fidelities exceeding $95 \%$ are achievable and are found to be mainly limited by motional dephasing and the finite lifetime of the Rydberg levels.
\end{abstract}

DOI: 10.1103/PhysRevLett.112.040501

Although optical photons are ideal for quantum communication, their utility for computation is limited by the lack of strong photon-photon interactions [1,2]. However, recently there has been substantial progress in this area using Rydberg ensembles [3-5], where the strong interactions between highly excited Rydberg atoms are mapped into strong interactions between individual optical photons [6-11]. In addition, quantum gate protocols based on Rydberg atoms have been proposed [12] and realized $[13,14]$ where the information was encoded in the ground state of the atoms instead of photons. The idea of exploiting the large dipole-dipole (DD) interactions between Rydberg atoms for photon processing has been analyzed theoretically for a variety of scenarios [15-18]. In general, the interaction is dissipative; however, dissipation can be reduced at the cost of interaction strength by detuning off resonance $[19,20]$. An additional problem is the implicit link between the interaction and propagation, which inevitably leads to a distortion of the photon wave packet and thereby precludes the realization of high-fidelity gates. In fact, it has been argued on fundamental grounds [21,22] that this problem occurs whenever conventional optical nonlinearities (such as cross-phase modulation) are used and cannot be circumvented.

In this Letter, we present a photon gate scheme that decouples light propagation and interaction, allowing the realization of high-fidelity photon-photon gates with negligible loss or distortion. We use the dark-state polariton protocol $[23,24]$ to convert two photonic qubits (control and target) in the dual rail encoding into collective excitations with Rydberg character in different positions or sites in an ensemble of cold atoms. We subsequently perform a $2 \pi$ rotation on the target qubit by using a microwave field

Published by the American Physical Society under the terms of the Creative Commons Attribution 3.0 License. Further distribution of this work must maintain attribution to the author(s) and the published articles title, journal citation, and DOI.
PACS numbers: 03.67.Lx, 32.80.Rm, 42.50.Ex, 71.36.+c

coupled to an auxiliary state, which by default gives an overall phase shift of $\pi$ radians to the qubit pair. The microwave field also induces resonant DD interactions [8] between the target and control sites that are closest together, preventing the rotation for one of the four qubit-pair states, and thereby implementing a controlled-z (CZ) phase gate. The excitations are then converted back to photons and emitted by the ensemble in the phase-matched direction.

Our scheme relies on the ability to modify the range of the DD interactions between highly excited Rydberg atoms using a resonant microwave field $[8,25,26]$. Using this field to couple to an auxiliary Rydberg state, we exploit the spatial independence of the dipole blockade mechanism [19] to induce a homogeneous phase shift on the stored photon and thereby circumvent the local-field limitation of the optical Kerr effect [21,22].

This Letter is organized as follows: First we outline the storage procedure, and then we show how, for two photons stored in adjacent sites in an atomic cloud, resonant DD interactions can be used to obtain a $\pi$ phase shift to the desired qubit state. Afterwards, we show that off-resonant, van der Waals (vdW) interaction between Rydberg levels in adjacent sites disrupts the ideal process of the gate but that its short-range effect can be overcome thanks to the long-range scaling of the resonant interactions. Finally, we give an estimate of the gate fidelity for the example of ultracold ${ }^{87} \mathrm{Rb}$ atoms, where we consider the effects of finite coupling strengths, extended spatial samples, and finite temperature.

The photonic qubit (for example, from a Rydberg singlephoton source [6]) is defined by using the dual-rail encoding [1], where the two states of the computational basis in each qubit $(|0\rangle$ and $|1\rangle)$ travel through two spatially separated regions of an atomic cloud or through two different clouds, although care should be taken to make the density and shape of each cloud as similar as possible to match the modes of the qubits. For a two-qubit gate, we consider four separate spatial paths (see Fig. 1). Similar geometries with two sites have already been implemented [13,14]. 


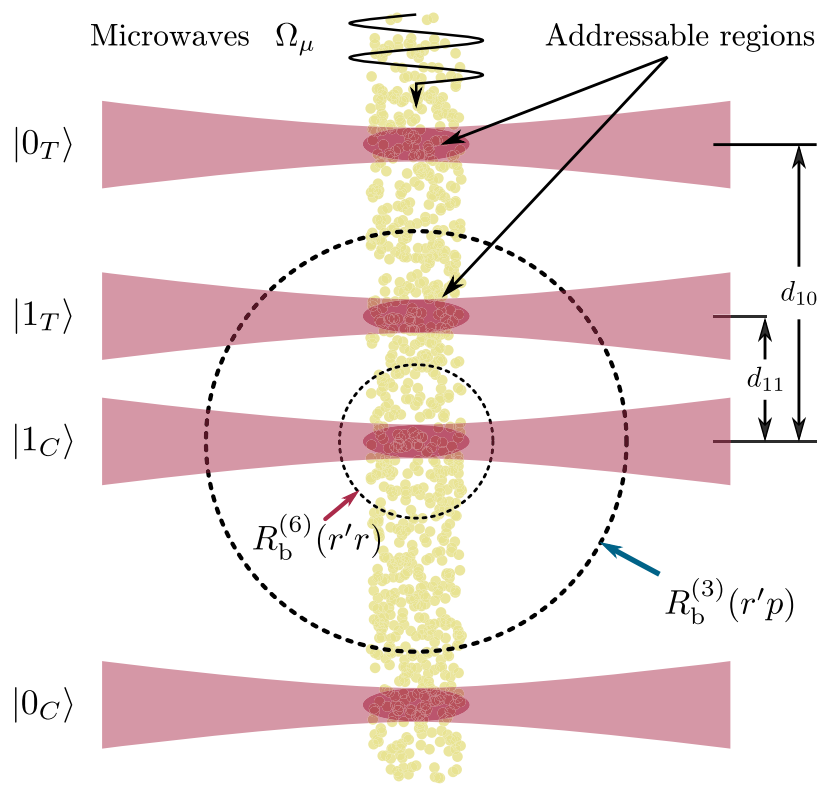

FIG. 1 (color online). Optical layout: Control $(|C\rangle)$ and target $(|T\rangle)$ photonic qubits, in dual-rail encoding, are stored as Rydberg polaritons (dark red) in a cold atomic ensemble (yellow). The spatial modes corresponding to the qubit states $\left|1_{C}\right\rangle$ and $\left|1_{T}\right\rangle$ are stored in adjacent sites at a distance $d_{11}$, and the others arbitrarily further apart from the interacting ones $\left(d_{10}\right)$. After storage we attempt a $2 \pi$ rotation on the target qubit using the microwave field with Rabi frequency $\Omega_{\mu}$ and an intermediate state. This succeeds except for $\left|1_{C} 1_{T}\right\rangle$, in which the intermediate state is shifted by resonant DD interactions, which have a characteristic length scale $R_{b}^{(3)}$. We need to ensure that the van der Waals interactions between stored states (characterized by the blockade radius $R_{b}^{(6)}$ ) are small.

We label the four channels as the elements of the set $\mathbb{B}=\left\{\left|1_{C}\right\rangle,\left|0_{C}\right\rangle,\left|1_{T}\right\rangle,\left|0_{T}\right\rangle\right\}$, where the subscripts represent the control $(C)$ and target $(T)$ qubits. We arrange the paths for the $|1\rangle$ (interacting) components to be adjacent, while the $|0\rangle$ paths are farther apart. We store the different photonic components in the medium as collective excitations (also called dark-state polaritons) with Rydberg character using electromagnetically induced transparency (EIT) in a ladder configuration $[4,7-10]$. To this end, the signal light is resonant with the closed atomic transition $|g\rangle \leftrightarrow|e\rangle$, and classical coupling lasers resonant with the transitions $|e\rangle \leftrightarrow|r\rangle$ or $|e\rangle \leftrightarrow\left|r^{\prime}\right\rangle$ are employed to store the control and target photons in two different Rydberg states $|r\rangle$ and $\left|r^{\prime}\right\rangle$ (see Fig. 2). These states can be of the form $|r\rangle=$ $|n L\rangle$ and $\left|r^{\prime}\right\rangle=\left|n^{\prime} L^{\prime}\right\rangle$, with $\left|L-L^{\prime}\right|=0,2$, but, to simplify, we use $|r\rangle=|n S\rangle$ and $\left|r^{\prime}\right\rangle=\left|n^{\prime} S\right\rangle$, where $n$ and $n^{\prime}$ are the principal quantum numbers and $S$ denotes the $L=$ 0 angular momentum state. Using different Rydberg states for target and control qubits allows us to perform operations on the individual qubits with a global microwave field.

There is at most one excitation in each site. This excitation is shared among all the atoms in that site, which maps the state into the superposition $\left|S_{j}\right\rangle=\left(1 / \sqrt{N_{j}}\right)$
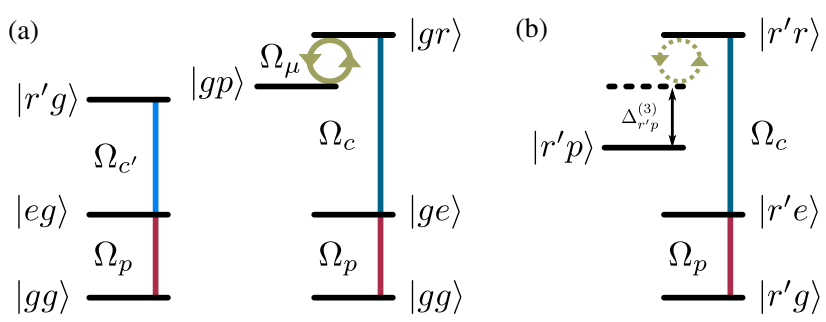

FIG. 2 (color online). Coupled basis of the two inner polariton sites $\left|1_{C} 1_{T}\right\rangle$. (a) The control (left) and target (right) photonic qubits are stored in the atom cloud in two different Rydberg states, $\left|r^{\prime}\right\rangle$ and $|r\rangle$, respectively. If the control qubit is in $|0\rangle$ (states $|g X\rangle$ in the pair state basis, with $X \in\{g, e, r, p\}$ ), we can perform a resonant $2 \pi$ rotation on the $|r\rangle \leftrightarrow|p\rangle$ transition. (b) If the control qubit is in $|1\rangle$ (states $\left|r^{\prime} X\right\rangle$ ), it shifts the auxiliary state $|p\rangle$ via a resonant DD interactions and the microwave $2 \pi$ pulse is no longer resonant. This gives rise to a homogeneous, conditional phase shift in the site.

$\sum_{k}^{N_{j}}\left|r_{k}^{j}\right\rangle e^{i \phi_{k}}$, where, at each site $j \in \mathbb{B}$ with $N_{j}$ atoms, the sum spans all possible singly excited states $\left|r_{k}^{j}\right\rangle$ to the Rydberg level $\left|r^{j}\right\rangle:|r\rangle$ in the target qubit and $\left|r^{\prime}\right\rangle$ in the control. The phase $\phi_{k}$ depends on the probe and coupling fields at the position of atom $k$. This process maps the photonic state $|C T\rangle=|C\rangle \otimes|T\rangle$ into a spin-wave state involving all of the four spatial channels $\left|S_{C T}\right\rangle=\left|S_{C}\right\rangle \otimes$ $\left|S_{T}\right\rangle$ and can be achieved with efficiencies per site exceeding $90 \%$ given sufficient optical depth [18] or by using a low-finesse optical cavity [27].

Once we have a mapping of the two-qubit state into the cloud, we make use of an auxiliary Rydberg state $|p\rangle$ in the target qubit to perform the gate operation. A microwave pulse is applied to the system to attempt a $\int_{0}^{t} \Omega_{\mu} d t=2 \pi$ rotation on the transition $|r\rangle \leftrightarrow|p\rangle$ in the target qubit, via the Hamiltonian $H_{\mu}=\hbar \Omega_{\mu}(|r\rangle\langle p|+| p\rangle\langle r|)$. Since there is only one excitation at each site, each ensemble behaves like an effective spin system, coupling the target states $\left|S_{T}\right\rangle$ and the superposition of singly excited $\left|p_{k}\right\rangle$ states, $\left|P_{T}\right\rangle=(1 / \sqrt{N}) \sum_{k}^{N_{C}}\left|p_{k}\right\rangle e^{i \phi_{k}}$, with the single-atom Rabi frequency $\Omega_{\mu}$. Since the wavelength of the microwave field is much greater than the separation between sites, the coupling to both target sites is the same.

Performing the $2 \pi$ rotation adds a $\pi$-phase shift to the wave function, $|C T\rangle \rightarrow-|C T\rangle$. However, if the target state $|p\rangle$ is coupled to the control Rydberg state $\left|r^{\prime}\right\rangle$ via an electric-dipole interaction at a distance $d$, DD interactions shift the energy of the coupled state $\left|r^{\prime} p\right\rangle$ by $\Delta_{r^{\prime} p}=C_{3}\left(r^{\prime} p\right) / d^{3}$, preventing the rotation and thus the phase shift, conditional on the presence of a control excitation in a nearby channel. This operation, which implements a CZ gate, occurs with arbitrarily high fidelity if the distance between the adjacent control and target channels, $d_{11}$, is smaller than the characteristic length:

$$
d_{11}<R_{b}^{(3)}\left(r^{\prime} p\right)=\sqrt[3]{C_{3}\left(r^{\prime} p\right) / \hbar \Omega_{\mu}}<d_{10}
$$


where $\Omega_{\mu}$ is the microwave Rabi frequency. The distance between noninteracting pairs, $d_{10}$, needs to be much larger than this length scale to prevent parasitic interactions.

However, the discussion outlined above is valid only if there are no other interactions between sites. If we have two excitations at a distance $d$ in the medium (one for each qubit), vdW interactions between the Rydberg levels $|r\rangle$ and $\left|r^{\prime}\right\rangle$ are important and can degrade the gate fidelity by introducing spatially dependent detunings. Such interactions detune the doubly excited state $\left|r^{\prime} r\right\rangle$ by an amount $\Delta_{r^{\prime} r}=C_{6}\left(r^{\prime} r\right) / d^{6}$ by coupling the states $\left|r^{\prime} r\right\rangle \leftrightarrow\left|p^{\prime} p\right\rangle$, where $|p\rangle$ and $\left|p^{\prime}\right\rangle$ are dipole-coupled to both $|r\rangle$ and $\left|r^{\prime}\right\rangle$. Here, $C_{6}\left(r^{\prime} r\right) \propto 1 / \delta_{f}$ is the vdW coefficient of an $\left|n^{\prime} S, n S\right\rangle$ pair state, where $\delta_{f}$ is the Förster energy defect $[28,29]$.

If the energy shift $\hbar \Delta_{r^{\prime} r}$ is comparable to the energy defect $\delta_{f}$, DD interactions populate neighbouring states. Also, if this energy defect is zero (a situation called Förster resonance), we expect excitation hopping between $\left|r^{\prime} r\right\rangle$ and $\left|p^{\prime} p\right\rangle$ to occur spontaneously. These scenarios can be avoided by choosing an appropriate level system. However, even for a system without Förster resonances, $\mathrm{vdW}$ interactions affect the proper functioning of the gate: during storage and retrieval processes and during the rotation in the microwave domain.

If we have an excitation $\left|r^{\prime}\right\rangle$ in one site, the interaction shift between sites prevents an excitation to $|r\rangle$ within a certain region characterized by the blockade length scale, $R_{b}^{(6)}\left(r^{\prime} r\right)=\left|C_{6}\left(r^{\prime} r\right) / \hbar \Omega\right|^{1 / 6}$, where $\Omega$ is the (powerbroadened) linewidth of the EIT transparency window. We minimize these intersite interactions by ensuring that the distance $d$ between any two spatial channels satisfies the inequality

$$
d>R_{b}^{(6)}\left(r r^{\prime}\right)
$$

This condition ensures that interactions during storage and retrieval are negligible, thus avoiding spatial distortion of the spatial modes of the qubit wave packets $[21,22]$.

The vdW interactions between $|r\rangle$ and $\left|r^{\prime}\right\rangle$ are present also during the microwave rotation, when the coupling laser is off. This space-dependent energy shift causes a dephasing to the state $|11\rangle$ proportional to the $2 \pi$ rotation time $\tau_{2 \pi}=$ $2 \pi / \Omega_{\mu}$ and to $\Delta_{r^{\prime} r}$. But it decreases rapidly with the distance between interacting sites and is negligible when

$$
d_{11}>R_{\mu}=\left|C_{6}\left(r^{\prime} r\right) / \hbar \Omega_{\mu}\right|^{1 / 6} .
$$

If we condition the interaction between $\left|1_{C}\right\rangle$ and $\left|1_{T}\right\rangle$ by (1) and make sure that the effects of vdW interactions are negligible during the storage and retrieval (2) and microwave rotation (3), the photonic component $|11\rangle$ picks up a homogeneous $\pi$ phase with respect to $|00\rangle,|10\rangle$, and $|01\rangle$, and the overall change in the system corresponds to that of a CZ gate [1].
Conditions (1), (2), and (3) suggest using $R_{b}^{(3)} /$ $\max \left(R_{b}^{(6)}, R_{\mu}\right)$ as the figure of merit to maximize the gate fidelity, but, in reality, both $R_{b}^{(3,6)}$ and $R_{\mu}$ are bounded by the shortest lifetime $\tau=1 / \Gamma$ in the Rydberg manifold. Instead, we use the dimensionless figure of merit (see Supplemental Material [30] for details)

$$
O=\left|\frac{C_{3}\left(r^{\prime} p\right)^{2}}{C_{6}\left(r^{\prime} r\right) \hbar \Gamma}\right| .
$$

Note that $O$ does not depend on any experimental parameters, only on the atomic species and the particular levels. Both $R_{b}^{(3,6)}$ and $O$ for ${ }^{87} \mathrm{Rb}$ are shown as a function of principal quantum number in Fig. 3.

To better understand the possible implementation of the phase gate including real-world sources of decoherence, we estimate the fidelity by using a simplified optical Blochequation approach. Our aim is not to provide a full many-body simulation of the gate protocol but rather to estimate the errors in the case of a physical realization by using a cloud of cold ${ }^{87} \mathrm{Rb}$ atoms. We shall note that we do not fully simulate storage and retrieval processes; instead, we use a one-photon transition to the Rydberg states to this effect (more details can be found in Supplemental Material [30]). We choose $\left|r^{\prime}\right\rangle=\left|n S_{1 / 2}\right\rangle$, $|r\rangle=\left|(n+1) S_{1 / 2}\right\rangle$, and $|p\rangle=\left|n P_{1 / 2}\right\rangle$ to maximize $O$ and avoid Förster resonances. For $n=70$, we obtain $R_{b}^{(6)} \sim$ $7 \mu \mathrm{m}$ and $R_{b}^{(3)} \sim 20 \mu \mathrm{m}$ by coupling to the $M=0$ state; i.e., the characteristic length of the resonant microwave transition is around 3 times larger than the optical blockade.

In Fig. 4, the results of this exploration are shown, where we have calculated the fidelity of the final state $\rho$, $F_{0}=\sqrt{\left\langle\psi^{\prime}|\rho| \psi^{\prime}\right\rangle}$, where the initial state $|\psi\rangle=$ $(|00\rangle+|01\rangle+|10\rangle+|11\rangle) / 2$ in the double-qubit basis
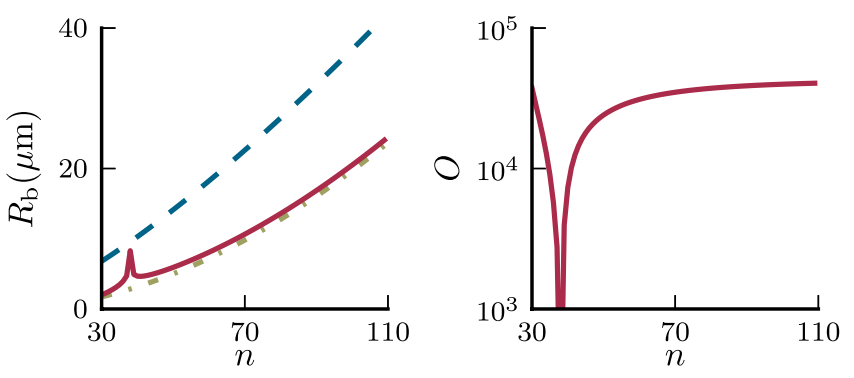

FIG. 3 (color online). (Left) The characteristic length scales $R_{b}$ as a function of principal quantum number $n$ for different pair states in ${ }^{87} \mathrm{Rb}$. The solid red line is the blockade radii for vdW interactions $R_{b}^{(6)}\left(n S_{1 / 2},(n+1) S_{1 / 2}\right)$. The dash-dotted, yellow line is the standard vdW blockade radii for a same-level pair state with coefficient $C_{6}\left(n S_{1 / 2}, n S_{1 / 2}\right)$ shown for reference. The dashed blue line is the long-range resonant interaction length scale $R_{b}^{(3)}\left(n S_{1 / 2}, n P_{1 / 2}\right)$ for the $M=0$ pair state. All radii are calculated for a coupling of $1 \mathrm{MHz}$. Note the Förster resonance for the coupling $38 s 39 s \leftrightarrow 38 p_{3 / 2} 38 p_{3 / 2}$ [31]. (Right) The figure of merit $O$ [see (4)] for $|n S\rangle,|(n+1) S\rangle$, and $|n P\rangle$ as a function of the principal quantum number $n$. 
becomes $\left|\psi^{\prime}\right\rangle=(|00\rangle+|01\rangle+|10\rangle-|11\rangle) / 2$ after the action of the gate. We initially obtain $F_{0}$ as a function of distance, keeping the rest of the parameters constant. To account for the finite width of the sites, we convolve $F_{0}$ with a Gaussian of width $w=\sqrt{2} q R_{b}^{(6)}\left(r^{\prime} r\right)$, where $q=$ $w_{0} / R_{b}^{(6)}\left(r^{\prime} r\right)$ is the ratio between the probe waist $w_{0}$ at each site and $R_{b}^{(6)}\left(r^{\prime} r\right)$. The $\sqrt{2}$ factor states that the interaction takes place between two sites. Finally, since the stored excitations are spin waves, and these can suffer from motional dephasing, we multiply the fidelity by a motional dephasing coefficient $\eta_{m} \propto \exp \left[-\left(t^{2} / \tau^{2}\right) /\left(1+t^{2} / \xi^{2}\right)\right]$ (taken from Ref. [32]), where atoms at a temperature $T$ and average speed $v=\sqrt{k_{B} T / m}$ ( $m$ is the atomic mass) can exit the site with mode diameter $w_{0}$ in a time $\xi=w_{0} / v$ or can move across the stored spin wave with wavelength $\Lambda$ in a time $\tau=\Lambda / 2 \pi v$. With these factors taken into account, we obtain processing fidelities over $95 \%$ over a broad range of experimental parameters (see Fig. 4).

Inspecting Fig. 4, we note the following general remarks: (a) Ensuring a strong coupling, and hence a large EIT

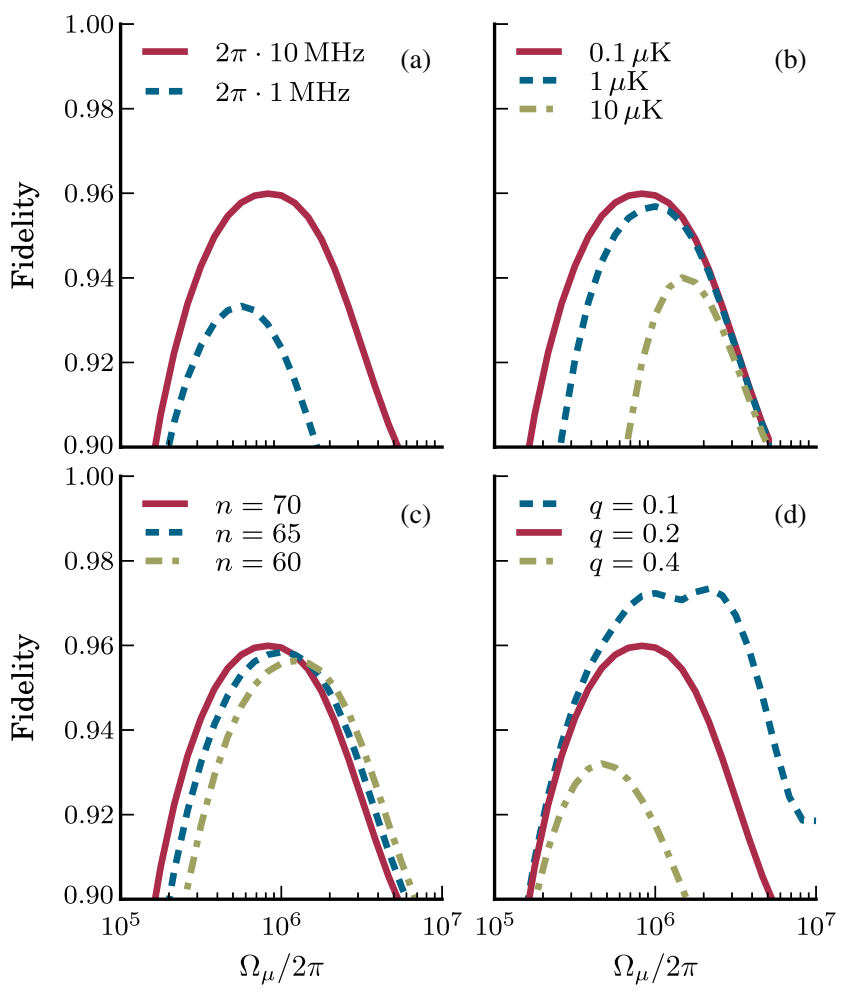

FIG. 4 (color online). Estimation of the fidelity of the gate protocol, including the effects of finite lifetimes of the Rydberg states and motional dephasing, as a function of the microwave Rabi frequency $\Omega_{\mu}$. The red, continuous line shows the case where $n=70, \Omega=2 \pi \times 10 \mathrm{MHz}$, and $q=0.2$ (see the text for details), for a temperature of $T=0.1 \mu \mathrm{K}$. Each plate shows the changes in the fidelity by varying one parameter: (a) EIT linewidth $\Omega$; (b) temperature $T$; (c) principal quantum number $n$; and (d) different waist to blockade ratios $q=w_{0} / R_{b}^{(6)}$ used in the Gaussian averaging. bandwidth $\Omega$ is key, as it allows the two interacting sites to be stored close together and profit from a higher resonant dipole shift. (c) Increasing the principal quantum number increases the fidelity, although we expect a weak scaling with $n$, as seen inspecting $O$ in Fig. 3. This happens because we can drive transitions in the microwave domain with a weaker $\Omega_{\mu}$ due to the favorable scaling of the lifetimes. However, this moves the peak of fidelity towards lower driving frequencies, making the gate operation slower, which puts this parameter in competition with motional dephasing. Finally, (d) the smaller the waist of the sites, the higher the fidelity, but this is limited by the diffraction limit; also, when the sites are very small, achieving a high optical depth (OD) is challenging and motional dephasing becomes a problem, although this could be reduced by using a state-insensitive optical lattice [33].

In addition to the limitations outlined above, a significant source of inefficiency is likely to arise from the mapping between the light field and the stored polaritons [18]. However, by making the cloud sufficiently dense $\left(N \sim 10^{14} \mathrm{~cm}^{-3}\right)$, it is possible to obtain optical depths OD $\sim 1000$ that would give an efficiency per channel of $\eta_{c} \approx 0.9$ and an overall [34] efficiency $\eta_{C}^{2} \approx 81 \%$, although denser samples might show more dephasing. This coupling efficiency can be further increased by using photonic waveguides or by optimizing the temporal shape of the probe pulse [18]. These numbers compare favorably with previous implementations using linear optics [35-37], which have a $1 / 9$ efficiency before postselection, and experimentally achieves $\eta^{2} \sim 85 \%$ after postselection. The process of storage and retrieval of polaritons with Rydberg content is still not fully understood, and further optimizations may be possible.

One can imagine using this scheme in combination with integrated chip atom trapping and waveguides [38] to join several quantum gates, both sequentially and in parallel. Using existing waveguide technology, one could also implement single qubit operations $[39,40]$ in the same chip, which brings us closer to a fully integrated quantum processor. Also, the proposed geometry and processing method could be extended to implement a photon switch and other operations.

In conclusion, we have shown that it is feasible to realize a quasideterministic, high-fidelity universal quantum gate for photons. We circumvent the restrictions of conventional optical nonlinearities by using the nonlocal dipole blockade effect and by separating the propagation and interaction phases of the gate. We exploit microwave fields to switch between short-range vdW interactions and longer-range resonant DD interactions, which allows us to achieve a conditional phase shift on the stored target photon with fidelities in excess of $95 \%$ for currently achievable experimental conditions. Nearly deterministic photon processing could facilitate a wide range of efficient quantum information protocols. 
We acknowledge financial support from Durham University, EPSRC grant reference EP/H002839/1 and the EU Marie Curie ITN COHERENCE Network. We thank H. Busche, D. Maxwell, D. J. Szwer, M. P. A. Jones, S. A. Gardiner, N. Henkel, and T. Gallagher for fruitful discussions and C. L. Vaillant for assistance with the DD interactions code.

*david.paredes@durham.ac.uk †c.s.adams@durham.ac.uk

[1] M. Nielsen and I. Chuang, Quantum Computation and Quantum Information, Cambridge Series on Information and the Natural Sciences (Cambridge University Press, Cambridge, England, 2000).

[2] S. Aaronson and A. Arkhipov, in Proceedings of the Fortythird Annual ACM Symposium on Theory of Computing, STOC '11 (Association for Computing Machinery, New York, 2011), pp. 333-342.

[3] M. Saffman, T. G. Walker, and K. Mølmer, Rev. Mod. Phys. 82, 2313 (2010).

[4] J. D. Pritchard, D. Maxwell, A. Gauguet, K. J. Weatherill, M. P. A. Jones, and C. S. Adams, Phys. Rev. Lett. 105, 193603 (2010)

[5] J. D. Pritchard, K. J. Weatherill, and C. S. Adams, in Annual Review of Cold Atoms and Molecules, edited by K. E. A. Madison (World Scientific, Singapore, 2013), pp. 301-350.

[6] Y. O. Dudin and A. Kuzmich, Science 336, 887 (2012).

[7] T. Peyronel, O. Firstenberg, Q.-Y. Liang, S. Hofferberth, A. V. Gorshkov, T. Pohl, M. D. Lukin, and V. Vuletić, Nature (London) 488, 57 (2012).

[8] D. Maxwell, D. J. Szwer, D. Paredes-Barato, H. Busche, J. D. Pritchard, A. Gauguet, K. J. Weatherill, M. P. A. Jones, and C. S. Adams, Phys. Rev. Lett. 110, 103001 (2013).

[9] C. S. Hofmann, G. Günter, H. Schempp, M. Robertde-Saint-Vincent, M. Gärttner, J. Evers, S. Whitlock, and M. Weidemüller, Phys. Rev. Lett. 110, 203601 (2013).

[10] S. Baur, D. Tiarks, G. Rempe, and S. Dürr, arXiv:1307.3509.

[11] O. Firstenberg, T. Peyronel, Q. Liang, A. Gorshkov, M. Lukin, and V. Vuletić, Nature (London) 502, 71 (2013).

[12] D. Jaksch, J. I. Cirac, P. Zoller, S. L. Rolston, R. Côté, and M. D. Lukin, Phys. Rev. Lett. 85, 2208 (2000).

[13] T. Wilk, A. Gaëtan, C. Evellin, J. Wolters, Y. Miroshnychenko, P. Grangier, and A. Browaeys, Phys. Rev. Lett. 104, 010502 (2010).

[14] L. Isenhower, E. Urban, X. L. Zhang, A. T. Gill, T. Henage, T. A. Johnson, T. G. Walker, and M. Saffman, Phys. Rev. Lett. 104, 010503 (2010).

[15] I. Friedler, D. Petrosyan, M. Fleischhauer, and G. Kurizki, Phys. Rev. A 72, 043803 (2005).

[16] E. Shahmoon, G. Kurizki, M. Fleischhauer, and D. Petrosyan, Phys. Rev. A 83, 033806 (2011).
[17] A. V. Gorshkov, J. Otterbach, M. Fleischhauer, T. Pohl, and M. D. Lukin, Phys. Rev. Lett. 107, 133602 (2011).

[18] A. V. Gorshkov, R. Nath, and T. Pohl, Phys. Rev. Lett. 110, 153601 (2013).

[19] S. Sevinçli, N. Henkel, C. Ates, and T. Pohl, Phys. Rev. Lett. 107, 153001 (2011).

[20] V. Parigi, E. Bimbard, J. Stanojevic, A. J. Hilliard, F. Nogrette, R. Tualle-Brouri, A. Ourjoumtsev, and P. Grangier,, Phys. Rev. Lett. 109, 233602 (2012).

[21] J. H. Shapiro, Phys. Rev. A 73, 062305 (2006).

[22] J. Gea-Banacloche, Phys. Rev. A 81, 043823 (2010).

[23] M. Fleischhauer and M. D. Lukin, Phys. Rev. Lett. 84, 5094 (2000).

[24] A. V. Gorshkov, A. André, M. D. Lukin, and A. S. Sørensen, Phys. Rev. A 76, 033805 (2007).

[25] M. Tanasittikosol, J. D. Pritchard, D. Maxwell, A. Gauguet, K. J. Weatherill, R. M. Potvliege, and C. S. Adams, J. Phys. B 44, 184020 (2011).

[26] F. Bariani, P. M. Goldbart, and T. A. B. Kennedy, Phys. Rev. A 86, 041802 (2012).

[27] E. Bimbard, R. Boddeda, N. Vitrant, A. Grankin, V. Parigi, J. Stanojevic, A. Ourjoumtsev, and P. Grangier, arXiv:1310.1228.

[28] D. Comparat and P. Pillet, J. Opt. Soc. Am. B 27, A208 (2010).

[29] C. L. Vaillant, M. P. A. Jones, and R. M. Potvliege, J. Phys. B 45, 135004 (2012).

[30] See Supplemental Material at http://link.aps.org/ supplemental/10.1103/PhysRevLett.112.040501 for a derivation of the figure of merit $O$ and a more detailed description of the estimation.

[31] J. Han and T.F. Gallagher, Phys. Rev. A 79, 053409 (2009).

[32] S. D. Jenkins, T. Zhang, and T. A. B. Kennedy, J. Phys. B 45, 124005 (2012).

[33] L. Li, Y. O. Dudin, and A. Kuzmich, Nature (London) 498 , 466 (2013).

[34] J. Nunn, K. Reim, K. C. Lee, V. O. Lorenz, B. J. Sussman, I. A. Walmsley, and D. Jaksch, Phys. Rev. Lett. 101, 260502 (2008).

[35] T. C. Ralph, N. K. Langford, T. B. Bell, and A. G. White, Phys. Rev. A 65, 062324 (2002).

[36] J. L. O’Brien, G. J. Pryde, A. G. White, T. C. Ralph, and D. Branning, Nature (London) 426, 264 (2003).

[37] M. A. Pooley, D. J. P. Ellis, R. B. Patel, A. J. Bennett, K. H. A. Chan, I. Farrer, D. A. Ritchie, and A. J. Shields, Appl. Phys. Lett. 100, 211103 (2012).

[38] M. Kohnen, M. Succo, P. G. Petrov, R. A. Nyman, M. Trupke, and E. A. Hinds, Nat. Photonics 5, 35 (2011).

[39] J. L. O'Brien and J. V. Akira Furusawa, Nat. Photonics 3, 687 (2009).

[40] A. Crespi, R. Ramponi, R. Osellame, L. Sansoni, I. Bongioanni, F. Sciarrino, G. Vallone, and P. Mataloni, Nat. Commun. 2, 566 (2011). 